\title{
Testicular adrenal rest tumours in congenital adrenal hyperplasia
}

\author{
H.L. Claahsen-van der Grinten, MD, PhD ${ }^{\mathrm{a}, *}$, B.J. Otten, MD, $\mathrm{PhD}^{\mathrm{a}}$, \\ M.M.L. Stikkelbroeck, MD, $\mathrm{PhD}^{\mathrm{b}}$, F.C.G.J. Sweep, MD, $\mathrm{PhD}^{\mathrm{c}}$, \\ A.R.M.M. Hermus, MD, $\mathrm{PhD}^{\mathrm{b}}$ \\ ${ }^{a}$ Department of Paediatric Endocrinology, Radboud University Nijmegen Medical Centre, The Netherlands \\ ${ }^{\mathrm{b}}$ Department of Endocrinology, Radboud University Nijmegen Medical Centre, The Netherlands \\ ${ }^{\mathrm{c}}$ Department of Chemical Endocrinology, Radboud University Nijmegen Medical Centre, The Netherlands
}

Keywords:

congenital adrenal hyperplasia testicular adrenal rest tumours infertility
In adult patients with congenital adrenal hyperplasia (CAH) the presence of testicular adrenal rest tumours (TART) is an important cause of gonadal dysfunction and infertility. In the last decade several papers have focused on the origin and pathogenesis of these tumours. In this paper we review the embryological, histological, biochemical and clinical features of TART and discuss the treatment options. Furthermore, we propose a new five-stage classification of TART, based on sonographic, clinical and biochemical parameters, that may lead to a better follow up and treatment of patients with TART.

(C) 2008 Elsevier Ltd. All rights reserved.

Congenital adrenal hyperplasia (CAH) is an inherited disorder of adrenal steroid synthesis. In more than $90 \%$ of cases it is caused by 21 -hydroxylase deficiency, leading to glucocorticoid deficiency and (in most cases) mineralocorticoid deficiency. ${ }^{1,2}$ The compensatory increase in adrenocorticotropic hormone (ACTH) secretion by the pituitary gland leads to stimulation of the adrenals and consequently overproduction of the adrenal androgen precursors dehydroepiandrosterone and androstenedione and subsequently of active androgens. The phenotype of 21-hydroxylase deficiency depends on the degree of enzyme deficiency. Treatment of 21-hydroxylase deficiency consists of glucocorticoid supplementation and in case of aldosterone deficiency also of mineralocorticoid replacement.

\footnotetext{
* Corresponding author. Department of Pediatric Endocrinology, Radboud University Nijmegen Medical Centre, (833), PO Box 9101, 6500 HB Nijmegen,

The Netherlands. Tel.: +31-24-3619118; Fax: +31-24-3668532.

E-mail address: h.claahsen@cukz.umcn.nl (H.L. Claahsen-van der Grinten).
} 
In the past, $\mathrm{CAH}$ was generally regarded as a paediatric endocrine disease, but nowadays nearly all patients reach adulthood as a result of improved diagnosis and treatment. One of the most serious problems in adult CAH patients is infertility. ${ }^{3-6}$ It is known that fertility is significantly decreased in male as well as in female CAH patients. ${ }^{4-15}$ The most important cause of male infertility in CAH patients is the presence of testicular tumours resulting in primary gonadal failure. These tumours were first reported in 1940 by Wilkins et al. ${ }^{16}$ Since then testicular tumours have been described in several papers, mainly as case reports. ${ }^{17-21}$ Because of the morphological and functional resemblance to adrenal tissue, as described later in this chapter, they are called testicular adrenal rest tumours (TART). They are always benign, although compression of the seminiferous tubules may lead to obstructive azoospermia, irreversible damage of the surrounding testicular tissue, and consequently infertility. Therefore, knowledge about the aetiology, pathogenesis and functional features of these tumours is important to develop adequate treatment strategies.

\section{Incidence}

The reported prevalence of TART in males varies between 0 and 94\%, dependent on the selection of the patients (age, hormonal control) and the method of tumour detection. ${ }^{4-8,22,23}$ As usually only tumours of $>2 \mathrm{~cm}$ are detectable by palpation because of their location within the rete testis, the tumours can be easily missed when additional imaging techniques such as ultrasound or magnetic resonance imaging (MRI) are not performed. Ultrasound and MRI are equally good methods for detection and monitoring of the tumours (Fig. 1), but ultrasound is preferable because it is quick and cheap $^{6,24}$, and even very small adrenal rests of only a few millimetres in diameter are detectable. In our own series, in 16 of the 17 patients (age 16-40 years) one or more testicular tumours were found. Most of them were not detectable by palpation. ${ }^{6}$

\section{Morphological aspects of TART}

Macroscopically, longstanding TART are firm and multilobular with a yellow to tan colour on cut surface and narrow bands of fibrous tissue (Fig. 2a). The typical location is within the rete testis, and in most patients the tumours are present bilaterally.

Histologically, TART resemble adrenocortical tissue. ${ }^{25,26}$ The tumours are sharply demarcated but not encapsulated, and consist of sheets or confluent cords of large polygonal cells with abundant eosinophilic cytoplasm, separated by dense fibrous tissue strands (Fig. 2b). Within the tumour fields there are regular thin fibrovascular septa, but a zonal arrangement is absent. The cytoplasm of the
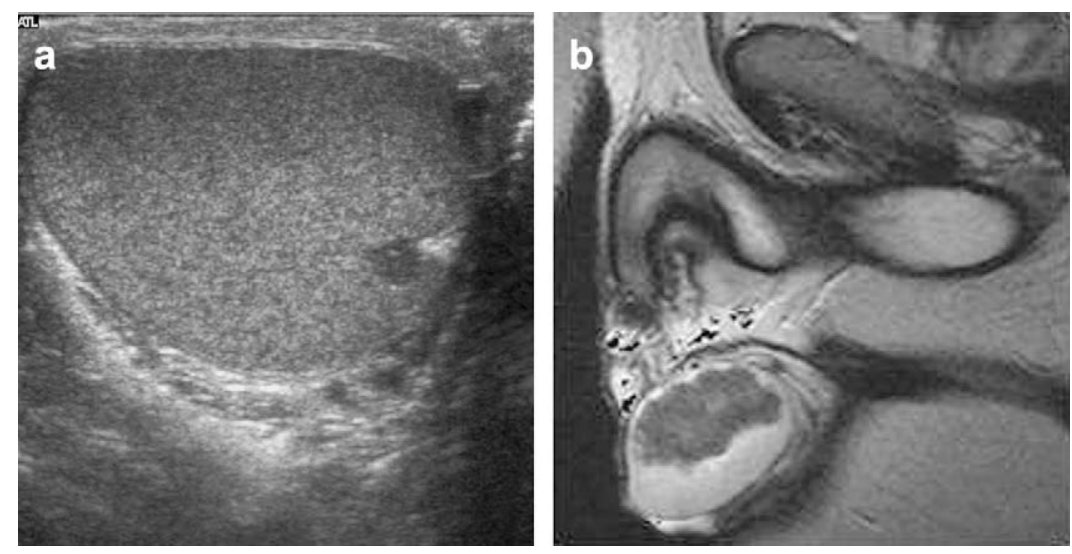

Fig. 1. (a) Scrotal ultrasound of a 13-year-old male CAH patient showing a mostly hypoechogenic rounded lesion in the left testis near the rete testis. (b) T2 weighted MR image of longstanding bilateral testicular adrenal rest tumour. Note that heterogeneous low-signal-intensity tumours are displacing surrounding high signal normal testicular tissue. 

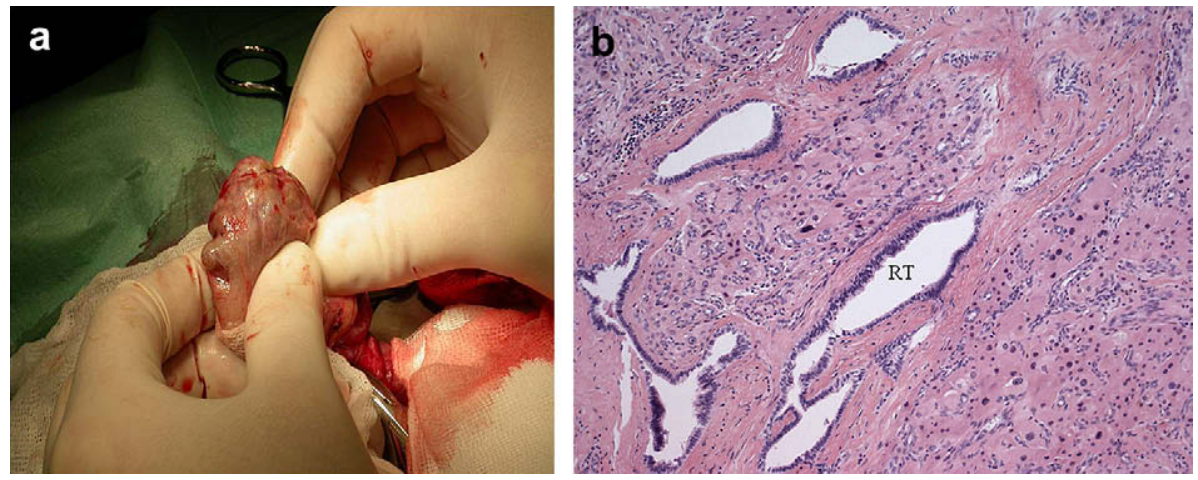

Fig. 2. (a) Macroscopic aspect of TART. Note the yellow colour and the bands of fibrous tissue. (b) Testicular adrenal rest tumour growing into rete testis $(\mathrm{RT})(\mathrm{HE}$, original magnification $\times 200)$.

tumour cells usually contains different amounts of lipofuscin pigment. The nuclei are round with a central prominent nucleolus and show clear variation in size with frequent intranuclear cytoplasmic inclusions. ${ }^{25,26}$

A clear histological differentiation between TART and malignant Leydig cell tumours is difficult. Some clinical features, however, can help to distinguish between these tumours: TART are bilateral in more than $80 \%$ of cases, whereas Leydig cell tumours are bilateral in only $3 \%$ of cases. Reinke crystals, which can be found in 25-40\% of Leydig cell tumours, are absent. Malignant degeneration is seen in $10 \%$ of Leydig cell tumours but have never been described in patients with TART. Furthermore, the typical location of the tumour in the rete testes can also help in the differentiation between these two types of tumours. 27,28

\section{The origin and pathogenesis of TART}

Several in vivo and in vitro studies, mainly in single patients, were performed to investigate the aetiology and the functional features of TART in CAH patients. Clark et al described the presence of the adrenal-specific enzyme CYP11B1 (11 $\beta$-hydroxylase) in tumour tissue of a single CAH patient with TART. ${ }^{29}$ Bercovici et al demonstrated the presence of adrenal-specific steroids in TART of one patient. ${ }^{30}$ The presence of adrenal-specific $11 \beta$-hydroxylated steroids such as 21 -deoxycorticosterone (21DB) and 21-deoxycortisol (21DF) in blood taken from the gonadal veins is reported in three single cases. ${ }^{30-32}$ This indicates the presence of adrenal-like tissue in the testes of CAH patients with 21-hydroxylase deficiency, because these steroids can only be synthesized by adrenal-specific 11-hydroxylation, without the need for the deficient 21-hydroxylation step.

In our own study of seven male CAH patients with bilateral TART undergoing testis-sparing surgery, we measured the concentrations of the adrenal-specific steroid 21DF and of 17-hydroxyprogesterone (17OHP) and androstenedione $(\mathrm{A})$ in blood taken from the spermatic veins during the operation. ${ }^{33}$ In addition, the mRNA expression of the adrenal-specific enzymes CYP11B1 and CYP11B2, as well as of ACTH and angiotensin II (AII) receptors in 16 testicular tumours of eight patients, was measured by quantitative polymerase chain reaction (PCR). We showed that TART contain adrenal-specific enzymes and produce adrenal-specific steroids, suggesting that these tumours arise from adrenal-like cells. The presence of ACTH and AII receptors at mRNA level in the adrenal rest tumours supports this hypothesis.

In the human adrenal gland CYP11B1 is expressed in high levels in the zona fasciculata/reticularis where it catalyses the $11 \beta$-hydroxylation of 11-deoxycortisol to cortisol. ${ }^{34}$ The presence of CYP11B1 in the zona glomerulosa is controversial. ${ }^{35}$ CYP11B2 is exclusively expressed in the zona glomerulosa where it is responsible for the final step of the aldosterone synthesis pathway. ${ }^{36,37}$ So, the presence of CYP11B1 and CYP11B2 in tumour tissue of all patients in our study group suggests that the tumours have functional features of both adrenal zona fasciculata and glomerulosa cells. 
It is known that AII has a strong trophic effect on the adrenal gland, especially on the zona glomerulosa. ${ }^{38-41}$ These trophic effects were studied in detail by Chatelain et al in adult rats showing that water deprivation resulted in high AII levels without affecting ACTH levels and in an increase of adrenal zona glomerulosa weight. ${ }^{38}$ Inhibition of AII production by angiotensin-converting enzyme inhibitors significantly decreased adrenal weight, suggesting that AII is an important factor in the stimulation of adrenal growth. AII markedly increases levels of both CYP11B1 and CYP11B2 mRNA, whereas ACTH causes an acute increase in CYP11B1 mRNA levels without an effect on CYP11B2 transcription. ${ }^{38}$ Therefore it can be speculated that tumour growth in $\mathrm{CAH}$ patients may not only be stimulated by elevated ACTH concentrations but also by elevated AII levels, as present in salt-wasting CAH patients with poor hormonal control. Interestingly, in late-onset CAH patients without clearly elevated ACTH or AII levels, testicular tumours have never been described.

\section{Embryology of TART}

The adrenal glands develop in the immediate vicinity of the gonads, and cells destined to become adrenocortical cells may nestle within the rete testis. ${ }^{42,43}$ In the literature a prevalence of up to $15 \%$ of such so-called adrenal rest cells in the testes of healthy neonates is reported. ${ }^{44,45}$ However, this prevalence is probably underestimated because single adrenal-like cells or small cell groups are very difficult to detect.

It is thought that poor hormonal control, leading to high blood levels of ACTH (and/or AII), is an important factor in the pathogenesis of TART inducing hypertrophy and hyperplasia of adrenal-like cells within the testis. ${ }^{19,29}$ However, TART are also found in adequately treated patients, whereas some poorly controlled male CAH patients never develop TART despite chronically elevated ACTH levels. 5,6 The most plausible explanation for this observation is that in the embryological period aberrant adrenal cells do not nestle in the testes in all males. The presence of these aberrant adrenal cells within the testis is a prerequisite for the development of TART, explaining the often observed discrepancy between the development of TART and hormonal control. It is likely that CAH patients without adrenal rest cells within their testes will never develop TART.

Our observation that TART can already be detected in early childhood, even in adequately treated patients, suggests that when adrenal rest cells are present within the testis even mildly or intermittently increased ACTH (and AII) concentrations may induce proliferation of these cells within the testis. Poor hormonal control with high ACTH levels may accelerate this process. It has to be realized that adrenal cells may already be stimulated in utero when there are elevated levels of ACTH.

So, both the concentrations of and the duration of exposure to growth-promoting factors are probably important in the pathogenesis of tumour growth. Furthermore, it can be hypothesized that the pubertal rise of luteinizing hormone ( $\mathrm{LH})$ may give an additional stimulation of tumour growth as LH receptors are found in TART ${ }^{46}$, which may explain the increased prevalence of TART in pubertal and post-pubertal CAH patients even when there is good hormonal control. Detailed studies focusing on the effects of ACTH, AII and LH in young male CAH patients are needed to determine the role of these factors in the development of TART.

The natural history of adrenal rest cells in healthy neonates is insufficiently known, but it is suggested that spontaneous regression occurs within the first years of life. ${ }^{5,6}$ It can be hypothesized that spontaneous regression of these adrenal rest cells will also occur in male CAH children when growthpromoting factors such as ACTH (and AII) are effectively suppressed in the first years of life. However, this treatment strategy conflicts with the negative effect of high doses of glucocorticoids on growth velocity in the first years of life. Therefore, the optimal medical treatment strategy in the first years of life is still not clear.

In a recent paper Val et al describe the presence of cells with mixed adrenal and Leydig cell properties within the mouse testis. ${ }^{47}$ These cells express adrenal markers such as CYP11B1 and CYP21 and respond to ACTH and human chorionic gonadotropin (HCG) incubation. These investigators hypothesize that adrenal rest cells may develop from a different population of adrenal-like cells. Of course these findings have to be translated with caution to the human population, as mouse fetal testes also express the ACTH receptor, in contrast to the developing human testes. ${ }^{47}$ 


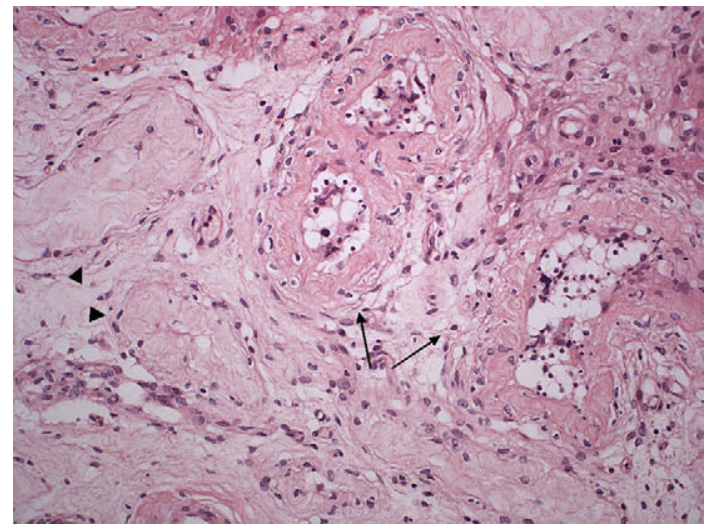

Fig. 3. Testicular biopsy of a patient showing seminiferous tubules with hypospermatogenesis and prominent peritubular fibrosis with increased number of peritubular fibroblasts (arrows), as well as tubular hyalinisation (arrow-head; original magnification $\times 200)$.

\section{Long-term consequences of TART}

TART have no malignant features, and therefore there seems to be no need to remove them at an early stage. However, because of the central localization of the tumours near the mediastinum testis, compression of the seminiferous tubules finally may lead to obstructive azoospermia and irreversible damage of the surrounding testicular tissue. In a recent study we showed a decreased tubular diameter and a varying degree of peritubular fibrosis and tubular hyalinization in the testicular biopsies of seven male CAH patients with longstanding bilateral TART and clinical infertility ${ }^{25}$ (Fig. 3). Furthermore, we found a severe decrease in the number of germ cells in all patients. In the literature, obstructive azoospermia is described mainly as a result of extra-testicular obstruction due to infections or surgical interventions mostly located at the epididymis or vas deferens. ${ }^{48-51}$ In these cases, adverse effects of the obstruction on the germinal epithelium or Leydig cells were not reported. ${ }^{49,51}$ This can be explained by the ability of the epididymis to become enlarged, to accommodate the sperm cells, and to phagocytose and resorb spermatozoa. ${ }^{49}$ It can be speculated that in the case of large TART located in the mediastinum testis proximal to the epididymis, the efferent flow in the seminiferous tubules is chronically obstructed without the possibility of compensatory dilatation of the epididymis. Longstanding obstruction of the seminiferous tubules could then lead to hypospermatogenesis and peritubular fibrosis.

In addition to the mechanical effects the tumours may also have a paracrine effect on the surrounding tissue. Steroids produced by the tumour cells may be toxic to the Leydig cells and/or germ cells. $^{52}$

The irreversible end-stage of longstanding TART is tubular hyalinization with obstruction of the lumen and complete loss of germ cells and Sertoli cells. In contrast to ischaemic hyalinization, where a reduced number of Leydig cells are expected, the interstitium of our patients contained a normal or only slightly reduced number of Leydig cells. ${ }^{25}$ Therefore, TART represent a very specific cause of obstructive azoospermia, commonly not mentioned in the literature, and with more severe clinical consequences than other forms.

\section{TART in childhood}

The presence of TART in children is described mostly in case reports ${ }^{53-56}$, and only a limited number of studies describe its prevalence in larger populations of children and adults. ${ }^{17,22,57}$ Avila et al detected TART by ultrasound in eight of 38 male CAH patients (age 6-31 years). ${ }^{22}$ The mean age of the patients was 14.8 years, and seven of the eight patients with TART were $<18$ years old. The youngest patient was 6.2 years old. The total number of investigated patients $<18$ years of age was not reported. Vanzulli et al 
described a prevalence of $27 \%$ of TART in a group of 30 CAH patients 9-32 years old. ${ }^{57}$ In the 24 investigated patients $<18$ years old, seven $(29 \%)$ had TART. However, these studies did not focus on childhood age and did not present information on gonadal function.

Shanklin et al studied autopsy material of patients with CAH and detected TART in three of seven patients $<8$ weeks old. ${ }^{17}$

In our own patient population we found that in 34 male CAH children (age 2-18 years) TART were already present in childhood with a prevalence of $24 \% .{ }^{59}$ The prevalence increased with age. None of the tumours was detectable by palpation, and none of the children with testicular tumours showed signs of gonadal dysfunction. In another study of 19 male CAH patients, age 2-10 years old, a similar prevalence of $21 \%$ was found. ${ }^{60}$ The investigators found significantly lower inhibin B values in the CAH group compared with a healthy control group, suggesting that gonadal dysfunction is already present in prepubertal $\mathrm{CAH}$ children. ${ }^{60}$

\section{Proposed classification of TART}

Based on the histological appearance of the TART and the surrounding testicular parenchyma, and the clinical observations described above, we propose that the development and growth of TART can be divided into five different stages (Fig. 4).

- Stage 1: This stage can be defined as the presence of adrenal rest cells within the rete testis, not detectable by scrotal ultrasound. In healthy boys these cells probably regress in utero or in the first years of life.

- Stage 2: In CAH patients, the adrenal rest cells may proliferate in the presence of increased concentrations of growth-promoting factors such as ACTH (and possibly also of AII). In this stage the adrenal rest cells may become visible by ultrasound as one or more small hypoechogenic lesions. The age of onset of cell growth may depend on the cumulative exposure to ACTH (and AII) concentrations over time and the number of ACTH (and AII) receptors on the adrenal rest cells.

- Stage 3: Further growth of the adrenal rest cells will compress the rete testis. In pubertal or postpubertal CAH patients, oligo- or azoospermia may already be found due to obstruction of the seminiferous tubules. Signs of gonadal dysfunction such as decreased inhibin B and increased follicle-stimulating hormone (FSH) and LH levels may also be present. At this stage tumour size may still be reduced by high dosages of glucocorticoids. However, because it is expected that tumour growth will restart after decreasing the dose of glucocorticoids, this is only a temporary solution.

- Stage 4: Further hypertrophy and hyperplasia of the adrenal rest cells with progressive obstruction of the rete testis may lead to induction of fibrosis within the tumour and focal lymphocytic infiltration. Several small tumours within the rete testis will conflate, forming a single lobulated structure separated from the residual testicular tissue by fibrous strands. In this stage high doses of glucocorticoids are probably no longer effective in decreasing tumour size because parts of the tumours contain fibrous tissue and/or because the adrenal rest cells may dedifferentiate in time with loss of ACTH and AII dependency. Furthermore, peritubular fibrosis can be found in the surrounding testicular tissue, indicating early testicular damage.

- Stage 5: Chronic obstruction subsequently will lead to destruction of the surrounding testicular parenchyma with irreversible damage of the testis.

Further studies are necessary to validate this proposed classification of TART.

\section{Diagnosis of TART}

Scrotal ultrasound and biochemical analysis may help to determine the presence and stage of TART in individual CAH patients. Because of their location within the rete testis, TART are difficult to palpate. Usually, only tumours with a size $>2 \mathrm{~cm}$ are detectable by palpation. Until now very small adrenal rests (stage 1) cannot be detected, not even with radiological techniques. Nowadays, ultrasound seems to be 
Stage 1

Adrenal rest cells present within the rete testis

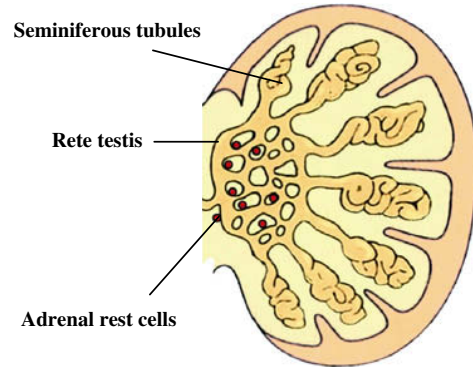

Stage 4

Induction of fibrosis and foca

lymphocytic infiltrates

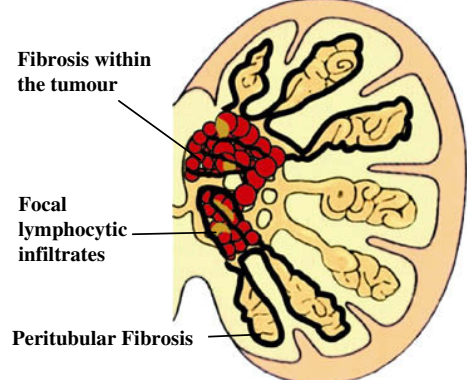

\section{Stage 2}

Hyperplasia and hypertrophy of adrenal rest cells

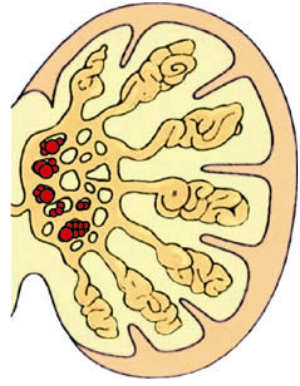

Stage 5

Irreversible damage of testicular parenchyma

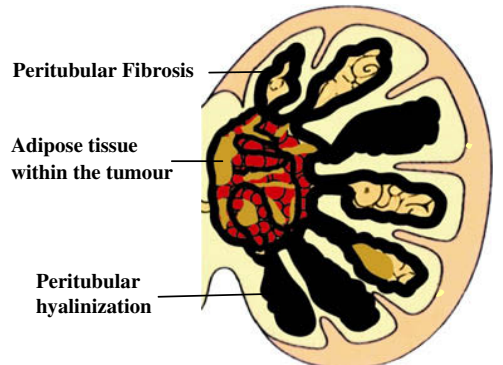

Stage 3

Further growth of the adrenal rest cells with compression of the rete testi

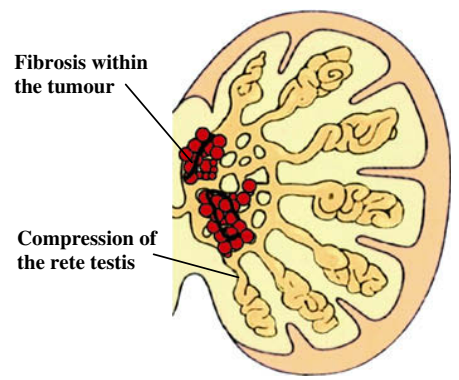


the best method for detection and follow-up, especially in the case of small non-palpable tumours. In stage 2 testicular tumours can be visible as small hypoechogenic lesions. From stage 3 onwards fibrous strands can be visible as hyperechogenic reflections.

Evaluation of gonadal function by determining blood LH, FSH, inhibin B and testosterone concentrations can help to determine the degree of gonadal failure as is expected from stage 3 onward. It should be realized that LH and FSH are of limited value to evaluate gonadal function in CAH patients because the gonadotrophins may be suppressed due to elevated adrenal androgens, which are partly aromatized to oestrone and oestradiol. ${ }^{61}$ In contrast to other causes of hypogonadotropic hypogonadism, CAH patients have generally normal or only slightly decreased testosterone levels because of elevated adrenal androgens. Inhibin B is a better marker for the evaluation of Sertoli cell function and may also be used in the evaluation of gonadal function in prepubertal children. ${ }^{62}$ Semen analysis can be performed in (post-)pubertal and adult patients when the patient is willing to collect semen for analysis.

Because tumour growth may be related to hormonal control as discussed earlier it is important to monitor plasma ACTH, renin, 17-hydroxyprogesterone and androstenedione concentrations.

In the case of longstanding tumours in infertile CAH patients, a testicular biopsy may be helpful to evaluate the quality of residual testicular parenchyma (stage 4 or 5 ). ${ }^{25}$ Such a biopsy is strongly advised before surgical treatment is offered. However, one should realize that a testicular biopsy only gives information about a limited area of the testis.

Because only patients in whom aberrant adrenal cells have been nestled in the testes in the embryological period are supposed to be at risk for developing TART, it would be very important to identify these patients as early as possible. Nowadays, adrenal rests can only be detected after substantial growth. In the future, new sensitive imaging techniques may help to detect these adrenal rests in the first years of life. If this is possible, patients with adrenal rests within their rete testis could be monitored and treated more intensively, whereas in patients without adrenal rests unnecessary ultrasound follow up and aggressive treatment strategies could be avoided.

\section{Treatment of TART}

The proposed classification of TART may help in the decision on which treatment option is useful in individual patients. TART stages 2 and 3 may be successfully treated by increasing the dose of glucocorticoids. Intensifying glucocorticoid therapy may lead to reduction of the tumour size by suppression of ACTH secretion, thereby improving testicular function. Although case reports with successful pregnancy of partners of male CAH patients have been published ${ }^{63}$, some studies report failure of intensified glucocorticoid treatment and serious side-effects after longstanding dexamethasone treatment, and therefore some patients will not accept this treatment option. ${ }^{18-20,63-65}$ Furthermore, it may be that this treatment leads only to temporary improvement of the obstruction because tumour growth may start again after lowering the glucocorticoid dose. However, optimizing glucocorticoid medication, especially in patients with poor hormonal control, is important to determine whether tumour growth is reversible (stage 3). Probably, in the future, new types of glucocorticoids - such as slow-release hydrocortisone or selective ACTH inhibitors - may help to suppress ACTH more effectively without the risk of adverse events. Because AII may also stimulate tumour growth the mineralocorticoid treatment has to be optimized.

In stage 4, increasing the dose of glucocorticoids is probably no longer effective in decreasing tumour size, but removal of the tumour may prevent further testicular damage. Because of the benign character of the tumours, testis-sparing surgery has been proposed for the treatment of TART. Walker et al performed testis-sparing surgery in three CAH patients. ${ }^{55}$ Postoperatively, there was good vascular flow and no recurrence of the tumour. Tiryaki et al reported two CAH patients with steroid-unresponsive testicular tumours, who were also treated by testis-sparing surgery. ${ }^{66}$ In neither study was information about pituitary-gonadal function before and after surgery reported. In a recent study we showed that in patients with longstanding TART (stage 5), gonadal dysfunction did not improve, suggesting irreversible damage to the surrounding testicular tissue. ${ }^{62}$ Furthermore, additional damage from surgery could not be excluded. From our experience we now 


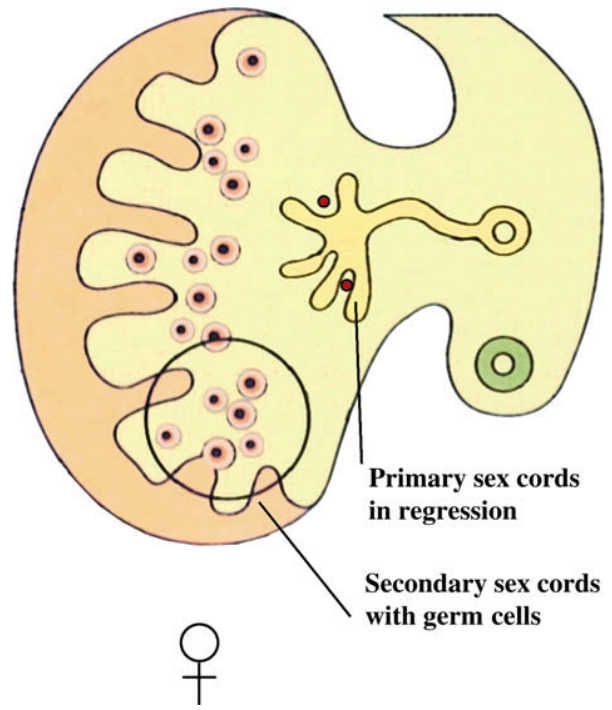

Fig. 5. Development of the female gonads. Note the presence of adrenal rest cells within the primary sex cords. It can be hypothesized that in the female gonad the adrenal rest cells will regress together with the primary sex cord.

conclude that in this stage the only indication for surgery is the relief of pain and discomfort caused by TART.

Therefore, mainly in longstanding TART with signs of gonadal dysfunction, testicular biopsies are advised to evaluate the quality of the surrounding testicular parenchyma before surgery is considered.

Further studies in childhood are needed to investigate whether surgery in stages 2, 3 and 4 may prevent irreversible damage to the testes. Hopefully, in order to prevent destruction of residual testicular parenchyma, introduction of new surgical techniques may facilitate the surgical treatment of the tumours already in childhood. As long as medical and surgical treatments of TART are far from perfect, patients should be informed about the negative effects of TART on fertility and cryopreservation of semen should be offered as soon as possible. Because adrenal rest cells are already present in the embryological period it is clear that prevention of TART is not possible.

\section{Ovarian adrenal rest tumours in female CAH patients}

Interestingly, in contrast to TART in male CAH patients, ovarian adrenal rest tumours in female CAH patients seem to be very rare and have been described in only three case reports. ${ }^{67-69}$ Stikkelbroeck et al searched for aberrant adrenal tissue in the gonads of female CAH patients with ultrasonography and MR. ${ }^{70}$ In none of the 13 female patients could ovarian adrenal rest tumours be detected.

To explain the striking difference between male and female CAH patients in the prevalence of gonadal adrenal rest tumours, we propose the following hypothesis: in the fifth week of the embryological period the gonadal ridge has the potential to develop into either a male or a female gonad, depending on the karyotype of the migrating primordial germ cells. In both male and female embryos the mesonephric duct forms primary sex cords (Fig. 5). In the male embryo these primary sex cords penetrate into the medulla of the developing testis, becoming the rete testis and seminiferous tubules. In these primary sex cords aberrant adrenal cells from the nearby developing adrenal cortex may easily nestle. In contrast, in the female embryo, the primary sex cords regress and secondary (cortical) sex cords will develop together with thickening of the surface epithelium. Therefore, in females aberrant adrenal cells nestled in the primary sex cords will also regress. 


\section{Practice points}

- TART is the most important cause of infertility in male CAH patients

- the incidence of TART in adult CAH patients, detected by ultrasound as the method of choice, is high (up to $94 \%$ )

- TART are also detectable in children with $\mathrm{CAH}$; gonadal dysfunction in childhood is controversial

- TART are not malignant, but longstanding TART can result in irreversible damage to testicular tissue

- TART have histological and functional features of adrenocortical tissue, and growth can be stimulated by elevated ACTH concentrations

- intensifying glucocorticoid therapy is the first step in the treatment of TART; before testissparing surgery is considered, testicular biopsies are advised to evaluate the quality of the surrounding testicular parenchyma

\section{Research agenda}

- evaluation of the natural history of TART and validation of the proposed classification

- early detection methods for TART

- role of angiotensin II and LH as growth-promoting factors

- medical and surgical treatment options

\section{References}

*1. Speiser PW \& White PC. Congenital adrenal hyperplasia. The New England Journal of Medicine 2003; 349: 776-788.

2. Forest MG. Recent advances in the diagnosis and management of congenital adrenal hyperplasia due to 21-hydroxylase deficiency. Human Reproduction Update 2004; 10: 469-485.

3. Wuesthof AR, Willig RP, Schulze W et al. Spermatogenesis is impaired in men with congenital adrenal hyperplasia. Hormone Research 1998; 50: 104.

4. Jaaskelainen J, Kiekara O, Hippelainen $\mathrm{M}$ et al. Pituitary gonadal axis and child rate in males with classical 21-hydroxylase deficiency. Journal of Endocrinological Investigation 2000; 23: 23-27.

5. Cabrera MS, Vogiatzi MG \& New MI. Long-term outcome in adult males with classic congenital adrenal hyperplasia. The Journal of Clinical Endocrinology and Metabolism 2001; 86: 3070-3078.

6. Stikkelbroeck NMML, Otten BJ, Pasic A et al. High prevalence of testicular adrenal rest tumours, impaired spermatogenesis, and Leydig cell failure in adolescent and adult males with congenital adrenal hyperplasia. The Journal of Clinical Endocrinology and Metabolism 2001; 86: 5721-5728.

7. Otten BJ, Stikkelbroeck NM, Claahsen-van der Grinten et al. Puberty and fertility in congenital adrenal hyperplasia. Endocrine Development 2005; 8: 54-66.

8. Urban MD, Lee PA \& Migeon CJ. Adult height and fertility in men with congenital virilizing adrenal hyperplasia. The New England Journal of Medicine 1978; 299: 1392-1396.

9. Stikkelbroeck NMML, Hermus ARMM, Braat DDM et al. Fertility in women with congenital adrenal hyperplasia due to 21hydroxylase deficiency. Obstetrical \& Gynecological Survey 2003; 58: 275-284.

10. Dumic M, Janjanin N, Ille J et al. Pregnancy outcomes in women with classical adrenal hyperplasia due to 21-hydroxylase deficiency. Journal of Pediatric Endocrinology \& Metabolism 2005; 18: 887-895.

11. Mulaikal RM, Migeon CJ \& Rock JA. Fertility rates in female patients with congenital adrenal hyperplasia due to 21hydroxylase deficiency. The New England Journal of Medicine 1987; 316: 178-182.

12. Hoepffner W, Schulze E \& Bennek J. Pregnancies in patients with congenital adrenal hyperplasia with complete or almost complete impairment of 21-hydroxylase deficiency. Fertility and Sterility 2004; 81: 1314-1320.

*13. Jaaskelainen J, Hippelainen M, Kiekara O et al. Child rate, pregnancy outcome and ovarian function in females with classical 21-hydroxylase deficiency. Acta Obstetricia et Gynecologica Scandinavica 2000; 79: 687-692.

14. Krone N, Wachter I, Stefanidou M et al. Mothers with congenital adrenal hyperplasia and their children: outcome of pregnancy, birth and childhood. Clinical Endocrinology 2001; 55: 523-529.

15. Lo JC \& Grumbach MM. Pregnancy outcomes in women with congenital virilizing adrenal hyperplasia. Endocrinology and Metabolism Clinics of North America 2001; 30: 207-229. IX.

16. Wilkins L, Fleishmann W \& Howard JE. Macrogenitosomia precox associated with hyperplasia of the androgenic tissue of the adrenal and death from corticoadrenal insufficiency. Endocrinology 1940; 26: 385-395. 
17. Shanklin DR, Richardson Jr. AP \& Rothstein G. Testicular hilar nodules in adrenogenital syndrome. American Journal of Diseases of Children 1963; 106: 243-250.

18. Rich MA, Keating MA, Levin HS et al. Tumours of the adrenogenital syndrome: an aggressive conservative approach. The Journal of Urology 1998; 160: 1838-1841.

*19. Bonaccorsi AC, Adler I \& Figueiredo JG. Male infertility due to congenital adrenal hyperplasia: testicular biopsy findings, hormonal evaluation, and therapeutic results in three patients. Fertility and Sterility 1987; 47: 664-670.

20. Giacaglia LR, Mendonca BB, Madureira G et al. Adrenal nodules in patients with congenital adrenal hyperplasia due to 21hydroxylase deficiency: regression after adequate hormonal control. Journal of Pediatric Endocrinology \& Metabolism 2001; 14: $415-419$.

*21. Rutgers JL, Young RH \& Scully RE. The testicular tumour of the adrenogenital syndrome. A report of six cases and review of the literature on testicular masses in patients with adrenocortical disorders. The American Journal of Surgical Pathology 1988; 12: 503-513.

22. Avila NA, Premkumar A, Shawker et al. Testicular adrenal rest tissue in congenital adrenal hyperplasia: findings at grayscale and colour Doppler US. Radiology 1996; 198: 99-104.

23. Avila NA, Premkumar A \& Merke DP. Testicular adrenal rest tissue in congenital adrenal hyperplasia: comparison of MR imaging and sonographic findings. AJR. American Journal of Roentgenology 1999; 172: 1003-1006.

24. Stikkelbroeck MML, Suliman HM, Otten BJ et al. Testicular adrenal rest tumours in postpubertal males with congenital adrenal hyperplasia: sonographic and MR features. European Radiology 2003; 13: 1597-1603.

*25. Claahsen-van der Grinten HL, Otten BJ, Hermus et al. Testicular adrenal rest tumours in patients with congenital adrenal hyperplasia can cause severe testicular damage. Fertility and Sterility 2008; 89: 597-601.

26. Rich MA \& Keating MA. Leydig cell tumours and tumours associated with congenital adrenal hyperplasia. Urologic Clinics of North America 2000; 27: 519-528.

27. Knudsen JL, Savage A \& Mobb GE. The testicular 'tumour' of adrenogenital syndrome - a persistent diagnostic pitfall. Histopathology 1991; 19: 468-470.

28. Newell ME, Lippe BM \& Ehrlich RM. Testis tumors associated with congenital adrenal hyperplasia: a continuing diagnostic and therapeutic dilemma. The Journal of Urology 1977; 117: 256-258.

*29. Clark RV, Albertson BD, Munabi A et al. Steroidogenic enzyme activities, morphology and receptor studies of a testicular adrenal rest in a patient with congenital adrenal hyperplasia. The Journal of Clinical Endocrinology and Metabolism 1990; 70: $1408-1413$.

30. Bercovici JP, Fiet J, Gibault L et al. Testicular adrenal rest tumours in salt wasting congenital adrenal hyperplasia (in vivo and in vitro studies). The Journal of Steroid Biochemistry and Molecular Biology 2005; 93: 67-72.

31. Combes-Moukhovsky ME, Kottler ML, Valensi P et al. Gonadal and adrenal catheterization during adrenal suppression and gonadal stimulation in a patient with bilateral testicular tumours and congenital adrenal hyperplasia. The Journal of Clinical Endocrinology and Metabolism 1994; 79: 1390-1394.

32. Blumberg-Tick J, Boudou P \& Nahoul K. Testicular tumors in congenital adrenal hyperplasia: steroid measurements from adrenal and spermatic veins. The Journal of Clinical Endocrinology and Metabolism 1991; 73: 1129-1133.

*33. Claahsen-van der Grinten HL, Otten BJ, Sweep CGJ et al. Testicular adrenal rest tumours in patients with congenital adrenal hyperplasia due to 21-hydroxylase deficiency show functional features of adrenocortical tissue. The Journal of Clinical Endocrinology and Metabolism 2007; 92: 3674-3680.

34. Rainey WE. Adrenal zonation: clues from $11 ß$ hydroxylase and aldosterone synthase. Molecular and Cellular Endocrinology 1999; 151: 151-160.

35. White PC, Curnow KM \& Pacoe L. Disorders of steroid 11ß hydroxylase isozymes. Endocrine Reviews 1994; 15: $421-438$.

36. Mornet E, Dupont J, Vitek A et al. Characterization of two genes encoding human steroid 11 beta-hydroxylase (P-450(11) beta). The Journal of Biological Chemistry 1989; 264: 20961-20967.

37. Curnow KM, Tusie-Luna MT, Pascoe R et al. The product of the CYP11B2 gene is required for the aldosterone biosynthesis in the human adrenal cortex. Molecular Endocrinology (Baltimore, Md.) 1991; 5: 1513-1522.

38. Chatelain D, Montel V, Dickes-Coopman A et al. Trophic and steroidogenic effects of water deprivation on the adrenal gland of the adult female rat. Regulatory Peptides 2003; 110: 249-255.

39. Mazzochi G, Rebuffat P, Robba $C$ et al. Trophic effects of potassium loading on the rat zona glomerulosa: permissive role of ACTH and angiotensin II. Acta Endocrinologica 1985; 108: 98-103.

40. McEwan PE, Vinson GP \& Kenyon CJ. Control of adrenal cell proliferation by AT1 receptors in response to angiotensin II and low-sodium diet. American Journal of Physiology. Endocrinology and Metabolism 1999; 276: 303-309.

41. Fallo F, Pezzi V, Barzon L et al. Quantitative assessment of CYP11B1 and CYP11B2 expression in aldosterone-producing adenomas. European Journal of Endocrinology 2002; 147: 795-802.

42. Mesinao S \& Jaffe RB. Developmental and functional biology of the primate fetal adrenal cortex. Endocrine Reviews 1997; 18: $378-403$.

43. Fujieda K \& Tajima T. Molecular basis of adrenal insufficiency. Pediatric Research 2005; 57: 62R-69R.

44. Sullivan JG, Gomel M \& Kinder RB. Ectopic adrenocortical tissue found at groin exploration in children: incidence in relation to diagnosis, age and sex. BJU International 2005; 95: 407-410.

45. Souverijns G, Peene P, Keuleers H et al. Ectopic localisation of adrenal cortex. European Radiology 2000; 10: 1165-1168.

46. Benvenga S, Smedile G, Lo GF et al. Testicular adrenal rests: evidence for luteinizing hormone receptors and for distinct types of testicular nodules differing for their autonomization. European Journal of Endocrinology 1999; 141: 231-237.

47. Val P, Jeays-Ward K \& Swain A. Identfication of a novel population of adrenal-like cells in the mammalian testis. Developmental Biology 2006; 299: 250-256.

48. Behre HM, Nieschlag E \& Meschede D. Obstructions of the seminal ducts. In Nieschlag E \& Behre H (eds.). Andrology. 2nd edn. Springer, 2001, pp. 179-181.

49. Wong TW \& Straus FH. Testicular biopsy in the study of male infertility. Archives of Pathology 1973; 95: 160-164.

50. Dondero F \& Lombardo F. Male infertility. In Wass JA \& Shalet SM (eds.). Oxford textbook of endocrinology and diabetes 2003.

51. Belmonte IG, Nistal M \& de Serrano M. Partial obstruction of the seminal path, a frequent cause of oligospermia in men. Human Reproduction 1998; 13: 3402-3405. 
52. Murphy H, George C, de Kretser D et al. Successful treatment with ICSI of infertility caused by azoospermia with adrenal rests in the testes. Human Reproduction 2001; 16: 263-267.

53. Boulware SD. Case report. An unusual case of precocious puberty. Current Opinion in Pediatrics 1997; 9: 443-446.

54. Erdogan S, Ergin M, Cevlik F et al. Testicular adrenal rest hyperplasia due to 21-hydroxylase deficiency: a case resport. Endocrine Pathology 2006; 17: 83-87.

*55. Walker BR, Skoog SJ, Winslow BH et al. Testis sparing surgery for steroid unresponsive testicular tumours of the adrenogenital syndrome. The Journal of Urology 1997; 157: 1460-1463.

56. Willi U, Atares M, Prader A et al. Testicular adrenal-like tissue (TALT) in congenital adrenal hyperplasia: detection by ultrasonography. Pediatric Radiology 1991; 21: 284-287.

57. Vanzulli A, Delmaschio A, Paesano P et al. Testicular masses in association with adrenogenital syndrome: US Findings. Radiology 1992; 183: 425-429.

*59. Claahsen-van der Grinten HL, Sweep FCGJ, Blickman JG et al. Prevalence of testicular adrenal rest tumours in male children with congenitaladrenal hyperplasia due to 21-hydroxylase deficiency. European Journal of Endocrinology 2007; 57: 339-344.

*60. Martinez-Aguayo A, Rocha A, Rojas C et al. Testicular adrenal rest tumors and Leydig and Sertoli cell function in boys with classical congenital adrenal hyperplasia. The Journal of Clinical Endocrinology and Metabolism 2007; 92: 4583-4589.

61. Claahsen-van der Grinten HL, Stikkelbroeck NM, Sweep CGJ et al. Fertility in patients with congenital adrenal hyperplasia. Journal of Pediatric Endocrinology \& Metabolism 2006; 19: 677-685.

62. Claahsen-van der Grinten HL, Otten BJ, Takahashi S et al. Testicular adrenal rest tumours in adult males with congenital adrenal hyperplasia: evaluation of pituitary-gonadal function before and after successful testis-sparing surgery in eight patients. The Journal of Clinical Endocrinology and Metabolism 2007; 92: 612-615.

63. Claahsen-van der Grinten, Otten BJ, Sweep CGJ et al. Repeated succesful induction of fertility after replacing hydrocortisone by dexamethasone in a patient with cogenital adrenal hyperplasia and testicular adrenal rest tumours. Fertility and Sterility 2007; 88. 705e5-705e8.

64. Cutfield R, Bateman JM \& Odell WD. Infertility caused by bilateral testicular masses secondary to congenital adrenal hyperplasia (21-hydroxylase deficiency). Fertility and Sterility 1983; 40: 809-814.

65. Stikkelbroeck NML, Hermus ARMM, Suliman HM et al. Asymptomatic testicular adrenal rest tumours in adolescent and adult males with congenital adrenal hyperplasia: basal and follow-up investigation after 2,6 years. Journal of Pediatric Endocrinology \& Metabolism 2004; 17: 6645-6653.

66. Tiryaki T, Aycan Z \& Huecumenglu S. Testis sparing surgery for steroid unresponsive testicular tumours of the congenital adrenal hyperplasia. Pediatric Surgery International 2005; 21: 853-855.

67. Russo G, Paesano P, Taccagni G et al. Ovarian adrenal-like tissue in congenital adrenal hyperplasia. The New England Journal of Medicine 1998; 339: 853-854.

68. Al Ahmadie HA, Stanek J, Liu J et al. Ovarian 'tumor' of the adrenogenital syndrome - the first reported case. The American Journal of Surgical Pathology 2001; 25: 1443-1450.

69. Claahsen-van der Grinten HL, Hulsbergen - van de Kaa CA \& Otten BJ. Ovarian adrenal rest tissue in congenital adrenal hyperplasia - a case report. Journal of Pediatric Endocrinology \& Metabolism 2006; 19: 177-182.

70. Stikkelbroeck MML, Hermus ARMM, Schouten D et al. Prevalence of ovarian adrenal rest tumors and polycystic ovaries in females with congenital adrenal hyperplasia: results of ultrasonography and MR imaging. European Radiology 2004; 14: 1802-1806. 